\title{
RNF213 p.R4810K Variant Carriers with Intracranial Arterial Stenosis Have a Low Atherosclerotic Burden
}

\author{
Mariko Ohara ${ }^{1}$, Takeshi Yoshimoto ${ }^{2}$, Shuhei Okazaki ${ }^{1,2}$, Yasufumi Gon ${ }^{1}$, Kenichi Todo ${ }^{1}$, Tsutomu Sasaki ${ }^{1}$, \\ Junji Takasugi ${ }^{3}$, Nobuyuki Ohara ${ }^{3}$, Masafumi Ihara ${ }^{2}$ and Hideki Mochizuki ${ }^{1}$
}

Mariko Ohara and Takeshi Yoshimoto contributed equally to this work.

${ }^{1}$ Department of Neurology, Osaka University Graduate School of Medicine, Osaka, Japan
${ }^{2}$ Department of Neurology, National Cerebral and Cardiovascular Center, Osaka, Japan
${ }^{3}$ Department of Neurology, Kobe City Medical Center General Hospital, Hyogo, Japan

Aim: The ring finger protein 213 gene (RNF213) p.R4810K variant is a major susceptibility gene for intracranial arterial stenosis in East Asia. We hypothesized that if intracranial arterial stenosis is induced by a non-atherosclerotic mechanism similar to moyamoya disease, the patients with RNF213 p.R4810K variant may have a lower cumulative atherosclerotic burden than the non-carriers.

Methods: A total of 112 participants with intracranial arterial stenosis were enrolled in this multicenter crosssectional study. We compared the prevalence of atherosclerotic risk factors and three different cardiovascular risk scores (Essen Stroke Risk Score, Framingham Risk Score, and Suita Risk Score) between the RNF213 p.R4810K variant carriers and non-carriers. Patients with moyamoya disease were excluded from the study.

Results: The RNF213 p.R4810K variant carriers were younger than the non-carriers $(P<0.001)$. The prevalence of each atherosclerotic risk factor was not significant, but it tended to be lower in the variant carriers. The Essen Stroke Risk Score (carriers: $2.3 \pm 1.5$ vs. non-carriers: $2.9 \pm 1.5, P=0.047$ ), Framingham Risk Score $(10.7 \pm 6.4$ vs. $15.3 \pm 6.2, P=0.001)$, and Suita Risk Score $(35.4 \pm 15.8$ vs. $48.7 \pm 15.2, P<0.001)$ were significantly lower in the variant carriers. Among the three risk scores, the Suita score showed the highest predictive accuracy for the variant carriers.

Conclusions: RNF213 p.R4810K variant carriers have a lower cumulative atherosclerotic burden than noncarriers among patients with intracranial arterial stenosis. New therapeutic approaches beyond the standard management of atherosclerotic risk factors are required to prevent the development of intracranial arterial stenosis.

Key words: RNF213, Risk factors, Atherosclerosis, Genetic polymorphism, Intracranial arteriosclerosis Non-standard Abbreviations and Acronyms: RNF213: Ring finger protein 213, CI: Confidence interval, OR: Odds ratio, ROC: Receiver operating curve, AUC: Area under the curve

\section{Introduction}

Intracranial arterial stenosis accounts for $30 \%-$ $50 \%$ of the causes of ischemic stroke in East Asia ${ }^{1,2)}$. Recently, the p.R4810K variant of the ring finger protein 213 gene (RNF213), which was identified as a susceptibility gene for moyamoya disease ${ }^{3,4)}$, was found to be a strong risk factor for intracranial arterial stenosis ${ }^{5,6)}$ and ischemic stroke ${ }^{7)}$. Approximately $22 \%-50 \%$ of patients with intracranial arterial stenosis reportedly have this variant ${ }^{5,6)}$, which is much higher than the prevalence in the general population

Address for correspondence: Shuhei Okazaki, Department of Neurology, Osaka University Graduate School of Medicine, 2-2 Yamadaoka, Suita, Osaka, 5650871, Japan E-mail: s-okazaki@umin.ac.jp

Received: November 18, $2021 \quad$ Accepted for publication: December 8, 2021

Copyright@2021 Japan Atherosclerosis Society

This article is distributed under the terms of the latest version of CC BY-NC-SA defined by the Creative Commons Attribution License. 


\section{$(1.3 \%-2.0 \%)^{7,8)}$.}

The mechanisms by which this variant causes intracranial arterial stenosis have not yet been elucidated, but it has been reported that the RNF213 protein is involved in caveolin-1-mediated endothelial cell function and angiogenesis9) and the variant carriers have smaller diameters of intracranial arteries, suggesting negative remodeling ${ }^{10,11)}$. On the other hand, positive remodeling is known to occur in response to atherosclerotic plaque formation ${ }^{12)}$. These findings suggest that intracranial arterial stenosis, generally thought to be caused by atherosclerosis, occurs via a different mechanism in variant carriers. We hypothesized that if intracranial arterial stenosis is induced by a non-atherosclerotic mechanism, RNF213 p.R4810K variant carriers may have a lower cumulative atherosclerotic burden than non-carriers.

\section{Aim}

In this study, we compared the prevalence of atherosclerotic risk factors between RNF213 p.R $4810 \mathrm{~K}$ variant carriers and non-carriers among patients with intracranial arterial stenosis. In addition, the cumulative atherosclerotic burden was estimated using three different cardiovascular risk scores and compared between the two groups. Because variant carriers have a higher rate of recurrent stroke ${ }^{13)}$, it is clinically important to identify the variant carriers in patients with intracranial arterial stenosis. Therefore, we examined the optimal cut-off values of each risk score for predicting the $R N F 213$ variant carriers.

\section{Methods}

The data that support the findings of this study are available from the corresponding author upon reasonable request.

\section{Study Design and Participants}

This multicenter cross-sectional observational study was conducted at three medical centers in Kansai district of Japan: Osaka University Hospital, Osaka; National Cerebral and Cardiovascular Center, Osaka; and Kobe City Medical Center General Hospital, Hyogo. Participants were recruited from these three medical centers. Written informed consent was obtained from all participants. This study was approved by the Ethics Committee for Clinical Research of Osaka University (approval number: 845) and each medical center. Patients who met the following criteria were included: (1) patients aged over 20 years; (2) patients with intracranial arterial stenosis defined as a diameter reduction of $\geq 50 \%$ or occlusion in the intracranial internal carotid artery, M1-M2 segment of the middle cerebral artery, A1-A2 segment of the anterior cerebral artery, P1-P2 segment of the posterior cerebral artery, or the basilar artery; and (3) available $R N F 213$ genetic test. We excluded patients diagnosed with intracranial arterial occlusion due to cardiogenic embolism or moyamoya disease according to the Japanese guidelines for moyamoya disease ${ }^{14)}$.

At Osaka University Hospital and Kobe City Medical Center General Hospital, 124 candidates with intracranial arterial stenosis were recruited from a prospectively collected database between January 2019 and February 2021. Among them, 13 patients diagnosed with moyamoya disease were excluded. Informed consent was obtained from 68 patients, and 2 were excluded because their blood samples were not available. At the National Cerebral and Cardiovascular Center, 4802 patients were admitted with acute ischemic stroke between January 2011 and December 2018. After providing their informed consent, 68 patients with intracranial arterial stenosis underwent RNF213 genotyping, and 22 patients diagnosed with cardioembolic stroke or moyamoya disease were excluded. Finally, 112 patients with intracranial arterial stenosis were enrolled in this study. Among the 112 enrolled patients, $11(9.8 \%)$ were included in the previous study ${ }^{7)}$.

\section{RNF213 Genotyping Examination}

Peripheral blood samples were obtained from all enrolled patients. Genotyping of RNF213 p.R $4810 \mathrm{~K}$ was performed using a fully automated gene analysis system (GTS-7000; Shimadzu Corporation, Kyoto, Japan or LightCycler 96 system; Roche, Basel, Switzerland). These systems allow the direct detection of single-nucleotide polymorphisms from $1 \mu \mathrm{L}$ of whole blood samples using real-time polymerase chain reaction ${ }^{15}$. The primer sequences used in the current analysis were 5'-TTCCAGAACGTCCAGCAAGT-3' (forward) and 5'-ACAGTCCTGGTCCTGTCAGA-3' (reverse). The probe sets used were 5' - C TCCATCAGAGGCTTCCT-3' and 5'-CTCCATCAAAGGCTTCCT-3'. As a result of $R N F 213$ p.R $4810 \mathrm{~K}$ variant genotype analysis, the GA and AA variants for p.R $4810 \mathrm{~K}$ were defined as variant carriers and the wild-type homozygous (GG) as noncarriers.

\section{Imaging Analysis}

The diagnosis of intracranial arterial stenosis was based on magnetic resonance angiography or computed tomography angiography findings. If a patient underwent more than one examination, the initial examination was used for the analysis. All

\section{Advance Publication Journal of Atherosclerosis and Thrombosis}


images were evaluated by an experienced neurologist (MO) who was blinded to the clinical information.

\section{Estimation of Cumulative Atherosclerotic Burden}

Patient characteristics, including age, sex, presence of atherosclerotic risk factors, history of cerebrovascular and cardiovascular diseases, blood pressure values, and laboratory data at the time of initial evaluation were extracted from a prospectively collected database and electronic medical records of each medical center. To estimate the cumulative arteriosclerotic burden, we calculated three different cardiovascular risk scores for each individual: 1) Essen Stroke Risk Score ${ }^{16)}$, 2) Framingham Risk Score ${ }^{17)}$, and 3) Suita Risk Score ${ }^{18)}$, as described in previous papers.

In brief, the Essen Stroke Risk Score is a tool for predicting the recurrence rate of stroke and combined cardiovascular events within 1 year, derived from a data subset from the Clopidogrel versus Aspirin in Patients at Risk of Ischemic Events trial ${ }^{19)}$. The score is calculated based on the presence or absence of eight risk factors (0-9 points): age, hypertension, diabetes mellitus, previous myocardial infarction, other cardiovascular disease, current smoking, peripheral arterial disease, and additional transient ischemic attack or ischemic stroke ${ }^{16)}$. The Framingham Risk Score is one of the most standard tools for assessing the 10-year risk of cardiovascular disease. The score is calculated as a function of age, sex, total cholesterol, high-density lipoprotein (HDL) cholesterol, systolic blood pressure, antihypertensive use, diabetes, and smoking habits (-5 to 31 points) ${ }^{17)}$. The Suita Risk Score is a new prediction algorithm for cardiovascular disease within 10 years based on a large urban cohort study in Japan ${ }^{20)}$, which is calculated based on the variables in the Framingham Risk Score (age, sex, total cholesterol, HDL cholesterol, blood pressure, diabetes, and smoking habit) and the severity of chronic kidney disease according to the estimated glomerular filtration rate (1-104 points) ${ }^{18)}$. Because data pertaining to the serum HDL cholesterol level and diastolic blood pressure were not available in one patient for each variable, mean imputation was used to calculate the Framingham Risk Score and Suita Risk Score.

\section{Statistical Analysis}

Categorical variables are presented as numbers and percentages and compared using the Chi-squared test. Continuous variables are presented as mean \pm standard deviation and compared using the $t$-test. Multivariate logistic regression analyses were used to calculate the adjusted odds ratios (ORs) and 95\% confidence intervals (CIs). Associations of atherosclerotic risk scores with the $R N F 213$ p.R $4810 \mathrm{~K}$ variant were analyzed by $t$-test. For clinical application, cut-off values for predicting the p.R $4810 \mathrm{~K}$ variant were determined from the receiver operating characteristic (ROC) curve using the Youden index. Statistical significance was defined as a $P$-value $<0.05$. A Bonferroni correction was applied for multiple comparisons in ROC analyses, with significance set at $P<0.016(0.05 / 3)$. All statistical analyses were performed using JMP Pro version 16.0.0 (SAS Institute Inc. Cary, NC, USA).

\section{Results}

\section{Patient Characteristics and RNF213 Variant}

The mean age of the 112 participants was $62.0 \pm$ 17.6 years, and $48 \%$ were female. No patient was homozygous for the AA genotype for p.R4810K, 30 $(27 \%)$ were heterozygous for the GA genotype, and $82(73 \%)$ were wild-type. Table 1 shows the characteristics of the carriers and non-carriers. The $R N F 213$ p.R $4810 \mathrm{~K}$ variant carriers were 11.9 years younger than the non-carriers $(P<0.001)$. The prevalence of atherosclerotic risk factors, such as hypertension, dyslipidemia, diabetes, and smoking habits, was not significantly different between the carriers and non-carriers. After adjusting for age and sex, there were no significant differences in the prevalence of atherosclerotic risk factors between the carriers and non-carriers. Similarly, blood pressure values, serum cholesterol levels, and estimated glomerular filtration rates were not significantly different between the two groups.

As shown in Table 1, all p.R $4810 \mathrm{~K}$ carriers and $96 \%$ of non-carriers had intracranial arterial stenosis or occlusion in the anterior circulation, whereas $20 \%$ of carriers and $24 \%$ of non-carriers had stenosis or occlusion in the posterior circulation. In comparison with the non-carriers, the variant carriers had a significantly higher prevalence of multiple intracranial arterial stenosis or occlusion (OR: 2.70, 95\% CI: $1.14-6.41, P=0.022)$. This significance was maintained after adjusting for age and sex.

\section{Cumulative Atherosclerotic Burden and RNF213 Variant}

Fig. 1 shows the distribution of the three different cardiovascular risk scores for p.R4810K carriers and non-carriers. Essen Stroke Risk Scores were significantly lower in carriers than in non-carriers $(2.3 \pm 1.5$ vs. $2.9 \pm 1.5, P=0.047)$. Similarly, Framingham Risk Scores and Suita Risk Scores were significantly lower in carriers $(10.7 \pm 6.4$ vs. $15.3 \pm 6.2$, $P=0.001 ; 35.4 \pm 15.8$ vs. $48.7 \pm 15.2, P<0.001$, 
Table 1. Patient characteristics of the RNF213 p.R4810K variant carriers and non-carriers

\begin{tabular}{|c|c|c|c|c|c|}
\hline & $\begin{array}{c}\text { Non carrier } \\
\qquad N=82\end{array}$ & $\begin{array}{l}\text { Carrier } \\
\mathrm{N}=30\end{array}$ & $P$ value & Odds ratio & Adj. odds ratio \\
\hline Age (year) & $65.2 \pm 17.1$ & $53.3 \pm 16.0$ & 0.001 & $0.96(0.93-0.99)$ & \\
\hline Hypertension & $64(78 \%)$ & $21(70 \%)$ & 0.378 & $0.66(0.26-1.68)$ & $0.57(0.17-1.85)$ \\
\hline Dyslipidemia & $56(68 \%)$ & $19(63 \%)$ & 0.621 & $0.80(0.33-1.93)$ & $0.98(0.38-2.51)$ \\
\hline Previous TIA or stroke & $35(43 \%)$ & $17(57 \%)$ & 0.189 & $1.76(0.75-4.09)$ & $0.75(0.31-1.86)$ \\
\hline Previous myocardial infarction & $7(9 \%)$ & $2(7 \%)$ & 0.747 & $0.77(0.15-3.91)$ & $0.66(0.12-3.80)$ \\
\hline Other cardiovascular disease & $12(12 \%)$ & $3(10 \%)$ & 0.735 & $0.79(0.21-3.02)$ & $0.62(0.14-2.65)$ \\
\hline Peripheral artery disease & $0(0 \%)$ & $0(0 \%)$ & & & \\
\hline Systolic blood pressure (mmHg) & $131.0 \pm 14.4$ & $128.2 \pm 17.3$ & 0.394 & $0.99(0.96-1.02)$ & $0.98(0.95-1.01)$ \\
\hline LDL-cholesterol (mmol/L) & $2.9 \pm 0.8$ & $2.8 \pm 0.8$ & 0.447 & $0.81(0.47-1.39)$ & $0.62(0.02-1.66)$ \\
\hline $\mathrm{eGFR}\left(\mathrm{ml} / \mathrm{min} / 1.73 \mathrm{~m}^{2}\right)$ & $68.2 \pm 22.6$ & $72.3 \pm 21.2$ & 0.381 & $1.01(0.99-1.03)$ & $0.99(0.96-1.01)$ \\
\hline Anterior circulation stenosis/occlusion & $79(96 \%)$ & $30(100 \%)$ & 0.288 & & \\
\hline Posterior circulation stenosis/occlusion & $20(24 \%)$ & $6(20 \%)$ & 0.626 & $0.78(0.28-2.16)$ & $1.23(0.40-3.73)$ \\
\hline Multiple stenosis/occlusion & $32(39 \%)$ & $19(63 \%)$ & 0.022 & $2.70(1.14-6.41)$ & $2.74(1.09-6.91)$ \\
\hline
\end{tabular}

TIA, transient ischemic attack; HDL, high-density lipoprotein; LDL, low-density lipoprotein; eGFR, estimated glomerular filtration rate; Adj. odds ratio, odds ratio adjusted for age and sex.

A

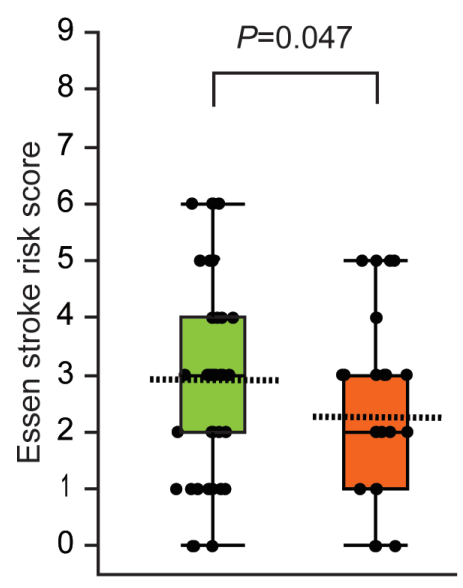

B

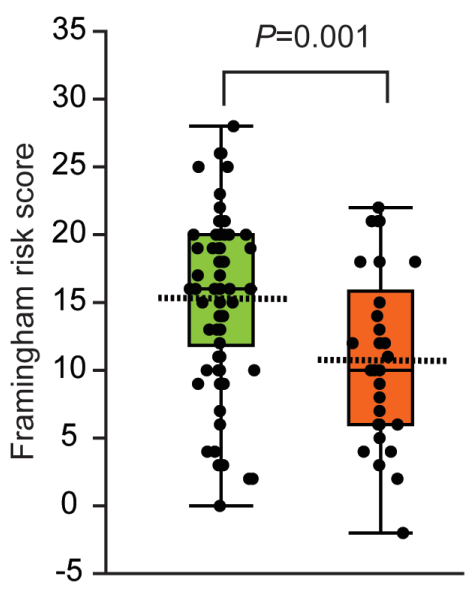

C

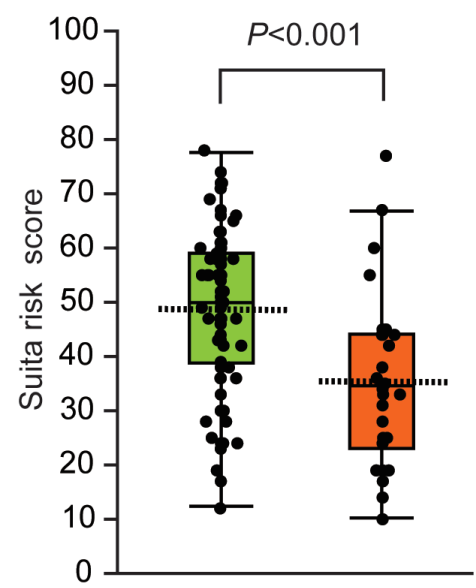

non-carrier $\square$ carrier

Fig. 1. Comparison of three cardiovascular risk scores between the RNF213 p.R $4810 \mathrm{~K}$ variant carriers and non-carriers

Essen Stroke Risk Score (A), Framingham Risk Score (B), and Suita Risk Score (C) were used to estimate the cumulative atherosclerotic burden. The variant carriers had a significantly lower cumulative atherosclerotic burden than the non-carriers in all three risk scores. Box plots indicate the 25th and 75th percentiles (box), median (black line), and mean value (dotted line), and whiskers extend to the upper and lower limits within 1.5 times the interquartile range from the box. 


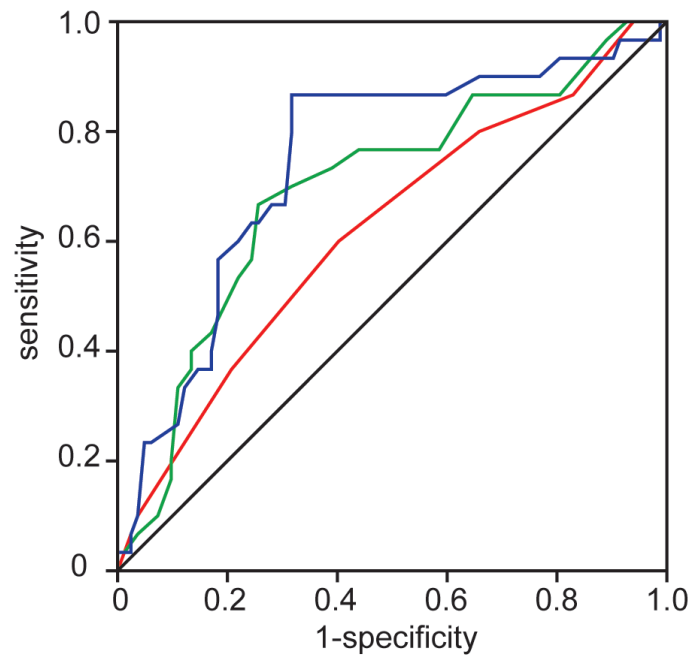

Essen stroke risk score

Framingham risk score

Suita risk score

Fig. 2. Receiver operating curves for predicting the $R N F 213$ p.R4810K variant carrier

The Suita Risk Score had the largest AUC (0.75), followed by the Framingham Risk Score (0.70) and Essen Stroke Risk Score (0.62).

Table 2. The optimal cut-off values of each risk score for predicting the $R N F 213$ variant carrier

\begin{tabular}{llccccc}
\hline & Range & Cut-off value & Sensitivity & Specificity & Odds ratio & Adj. odds ratio \\
\hline Essen stroke risk score & {$[0,6]$} & 2 & 0.60 & 0.60 & $2.23(0.95-5.23)$ & $0.74(0.22-2.53)$ \\
Framingham risk score & {$[-2,28]$} & 12 & 0.67 & 0.74 & $5.81(2.35-14.39)$ & $10.35(1.96-54.55)$ \\
Suita risk score & {$[10,78]$} & 45 & 0.87 & 0.68 & $14.00(4.43-44.25)$ & $20.19(4.25-95.96)$ \\
\hline
\end{tabular}

Range indicates the maximum and minimum values in this population. Adj. odds ratio indicates odds ratio adjusted for age, sex, and the presence of multiple intracranial arterial stenosis.

respectively).

To identify the impact of age on risk scores, we first performed sensitivity analyses by setting the age score to 0 for each risk score. The Essen Risk Score showed no significant difference between the carriers and non-carriers $(1.9 \pm 1.2$ vs. $2.0 \pm 1.2, P=0.836)$. The Framingham Risk Score was marginally lower in carriers $(3.8 \pm 3.7$ vs. $5.2 \pm 3.5, P=0.076)$ than in noncarriers. Meanwhile, the Suita Risk Score was significantly lower in carriers $(-5.4 \pm 10.0$ vs. $-1.4 \pm$ $8.7, P=0.040)$ even after disregarding the score of age. Next, because of the uneven distribution of variant carriers in the young population, we excluded patients under 50 years of age $(n=33)$ from the analyses. The Framingham Risk Score and Suita Risk Score were still significantly lower in carriers $(15.3 \pm 5.0$ vs. $17.8 \pm 4.2, P=0.046 ; 46.6 \pm 13.2$ vs. $54.9 \pm 9.8$, $P=0.007$, respectively), but there was no difference in the Essen Stroke Risk Score between the two groups $(3.3 \pm 1.3$ vs. $3.3 \pm 1.4, P=0.990)$.

\section{ROC Analysis}

The ROC curves and area under the curve (AUC) for each risk score are shown in Fig. 2. The largest AUC was obtained for the Suita Risk Score (0.75), followed by the Framingham Risk Score (0.70) and the Essen Stroke Risk Score (0.62). The AUC of the Suita Risk Score was significantly larger than that of the Essen Stroke Risk Score $(P=0.002)$ and tended to be larger than that of the Framingham Risk Score $(P=0.066)$. The optimal cut-off value for predicting the RNF213 p.R $4810 \mathrm{~K}$ variant carrier was 2 for the Essen Stroke Risk Score (sensitivity, 0.60; specificity, 0.60), 12 for the Framingham Risk Score (sensitivity, 0.67; specificity, 0.74), and 45 for the Suita Risk Score (sensitivity, 0.87; specificity 0.68) (Table 2).

When a Suita Risk Score of $\leq 45$ points was adopted as the cut-off point, the OR for predicting the variant carrier was 14.00 (95\% CI: 4.43-44.25; Table 2). In a multivariate logistic regression model where age, sex, the presence of multiple stenosis, and 
Suita Risk Score of $\leq 45$ points were included, only the Suita Risk Score of $\leq 45$ points was an independent predictor of the RNF213 p.R $4810 \mathrm{~K}$ variant (adjusted OR: 20.19, 95\% CI: 4.25-95.96, $P$ $<0.001)$.

\section{Discussion}

The present study showed that all three different cardiovascular risk scores were significantly lower in the RNF213 p.R $4810 \mathrm{~K}$ variant carriers than in noncarriers, yet the prevalence of each atherosclerotic risk factor was not significantly different between the two groups. Among the three risk scores, the Suita Risk Score showed the highest predictive accuracy for the variant carriers, and a Suita Risk Score of $\leq 45$ points was an independent predictor of the variant carriers. These results suggest that the variant carriers had a lower cumulative atherosclerotic burden than the noncarriers, and that the Suita Risk Score can be a useful tool to screen variant carriers in East Asian patients with intracranial arterial stenosis.

In this study, the p.R $4810 \mathrm{~K}$ variant carriers were younger and included more females than non-carriers. The prevalence of multiple intracranial stenosis was higher in the carriers. These results are consistent with those of previous studies ${ }^{7,13,21)}$. Similar to the present study, it was reported that the prevalence of atherosclerotic risk factors, such as hypertension, dyslipidemia, and diabetes, was slightly lower in the carriers, but the difference was not significant ${ }^{13,21)}$. It is recognized that each single risk factor, such as hypertension and dyslipidemia, has a small effect on the development of atherosclerosis, but the combined effect of multiple risk factors can additively increase the risk ${ }^{22}$. It is important to evaluate the cumulative atherosclerotic burden to assess the individual risk of cardiovascular disease. We used the three risk scores in this study. The Essen Stroke Risk Score, developed in Europe, adopts a simple and easy scoring system, in which all risk factors are scored in a binary manner (1, present; 0 , absent), and the severity is not considered. Therefore, the predictive accuracy may be inferior to the other two scores. The Framingham Risk Score, which was developed in the United States, is the most popular tool for assessing the cardiovascular risk. However, it has been reported that the Framingham Risk Score tends to overestimate the risk of cardiovascular disease in the Asian population ${ }^{23)}$ because the absolute risk of cardiovascular events is much lower in Asia ${ }^{24)}$. In Asian population studies, chronic kidney disease has been reported to be an independent risk factor for cardiovascular disease ${ }^{25)}$. Based on these characteristics in Asia, the Suita Risk
Score was developed to accurately predict the risk of cardiovascular disease in the Asian population ${ }^{18)}$. In this study performed in Japan, the Suita Risk Score showed the highest predictive accuracy among the three risk scores. Regional characteristics are likely to contribute to these differences among the three risk scores.

Because all three risk scores are strongly affected by age, we performed sensitivity analyses using two different approaches to evaluate the influence of age. The Suita Risk Score was significantly lower, and the Framingham Risk Score was marginally lower in the variant carriers, even after disregarding the influence of age. Multivariate logistic analysis also showed that the Suita Risk Score was an independent predictor of variant carriers even after adjusting for age and sex. A large Japanese population study ${ }^{26}$ showed that the mean value of the Suita Risk Score in the general population was approximately 43, which is higher than that of 35.4 in the variant carriers with intracranial stenosis. Because the average age was different between the two populations, it is not possible to simply compare these two values; however, these results indicate that the RNF213 p.R $4810 \mathrm{~K}$ variant carriers develop intracranial arterial stenosis even if they have a lower cumulative atherosclerotic risk than the general population.

Two possible mechanisms were considered for the development of intracranial arterial stenosis in variant carriers. First, the RNF213 variant enhances susceptibility to vessel wall injury, and the accumulation of minor atherosclerotic risks accelerates intracranial atherosclerosis under the condition of vascular vulnerability. In this case, more aggressive management of atherosclerotic risk factors from the early stage may reduce the development and progression of intracranial stenosis in variant carriers. Another hypothesis is that a non-atherosclerotic mechanism causes intracranial vessel narrowing independent of conventional atherosclerotic risk factors, similar to that of child-onset moyamoya disease. Our results appear to support the second hypothesis; however, further comparisons of longterm prognosis between RNF213 variant carriers with and without a cumulative atherosclerotic burden are necessary to elucidate the exact mechanisms. Recently, it has been found that RNF213 affects vascular endothelial cell functions and angiogenesis through caveolin-1 and NFAT1 ${ }^{9}$, 27). RNF213 also influences fat metabolism ${ }^{28)}$ and inflammation via the NF-kB pathway ${ }^{29)}$. Research on further approaches to these non-atherosclerotic pathways may lead to the development of new treatments for intracranial arterial stenosis.

\section{Advance Publication Journal of Atherosclerosis and Thrombosis}


This study had several limitations. First, because clinical information was obtained from previous medical records, the prevalence of atherosclerosis risks can be underestimated because of insufficient diagnostic surveillance. Second, sensitivity analyses were performed to remove the influence of age on risk scores, but this might be insufficient because some parameters, such as blood pressure and estimated glomerular filtration rate, are also affected by age. Third, because only Japanese patients were enrolled in this study, it is not clear whether these results can be adopted in other countries. Further multinational studies, especially in East Asia, where many asymptomatic carriers of the variant live, are warranted to verify these results.

In conclusion, this study showed that the RNF213 p.R $4810 \mathrm{~K}$ variant carriers had a lower cumulative atherosclerotic burden than the noncarriers among patients with intracranial arterial stenosis. Our results suggest that new therapeutic approaches beyond the standard management of atherosclerotic risk factors are required to prevent the development of intracranial arterial stenosis.

\section{Acknowledgements}

We are grateful to Dr.Yumi Yamamoto and Ms. Natsuki Hanada for technical assistance of RNF213 genotyping.

\section{Notice of Grant Support}

This work was supported by JSPS KAKENHI (Grant Number: JP19K06922) and the Japan Agency for Medical Research and Development (Grant Number: 21ek0210120h0003).

\section{$\mathrm{COI}$}

This study has no conflicts of interest to disclosure.

\section{References}

1) Wong LKS: Global burden of intracranial atherosclerosis. Int J Stroke, 2006; 1: 158-159

2) Hongo $H$, Miyawaki $S$, Imai $H$, Shimizu $M$, Yagi $S$, Mitsui J, Ishiura H, Yoshimura J, Doi K, Qu W, Teranishi Y, Okano A, Ono H, Nakatomi H, Shimizu T, Morishita $S$, Tsuji S, Saito N: Comprehensive investigation of RNF213 nonsynonymous variants associated with intracranial artery stenosis. Sci Rep, 2020; 10: 11942

3) Liu W, Morito D, Takashima S, Mineharu Y, Kobayashi $\mathrm{H}$, Hitomi T, Hashikata H, Matsuura N, Yamazaki S, Toyoda A, Kikuta K, Takagi Y, Harada KH, Fujiyama A,
Herzig R, Krischek B, Zou L, Kim JE, Kitakaze M, Miyamoto S, Nagata K, Hashimoto N, Koizumi A: Identification of RNF213 as a susceptibility gene for moyamoya disease and its possible role in vascular development. PLoS One, 2011; 6: e22542

4) Kamada F, Aoki Y, Narisawa A, Abe Y, Komatsuzaki S, Kikuchi A, Kanno J, Niihori T, Ono M, Ishii N, Owada Y, Fujimura M, Mashimo Y, Suzuki Y, Hata A, Tsuchiya S, Tominaga T, Matsubara Y, Kure S: A genome-wide association study identifies RNF213 as the first Moyamoya disease gene. J Hum Genet, 2011; 56: 34-40

5) Miyawaki S, Imai H, Takayanagi S, Mukasa A, Nakatomi $\mathrm{H}$, Saito N: Identification of a genetic variant common to moyamoya disease and intracranial major artery stenosis/ occlusion. Stroke, 2012; 43: 3371-3374

6) Bang OY, Ryoo S, Kim SJ, Yoon CH, Cha J, Yeon JY, Kim KH, Kim G-M, Chung C-S, Lee KH, Shin HJ, Ki C-S, Jeon P, Kim J-S, Hong SC: Adult moyamoya disease: A burden of intracranial stenosis in East Asians? PLoS One, 2015; 10: e0130663

7) Okazaki S, Morimoto T, Kamatani Y, Kamimura T, Kobayashi H, Harada K, Tomita T, Higashiyama A, Takahashi JC, Nakagawara J, Koga M, Toyoda K, Washida K, Saito S, Takahashi A, Hirata M, Matsuda K, Mochizuki H, Chong M, Paré G, O’Donnell M, Ago T, Hata J, Ninomiya T, Dichgans M, Debette S, Kubo M, Koizumi A, Ihara M: Moyamoya disease susceptibility variant RNF213 p.R4810K increases the risk of ischemic stroke attributable to large-artery atherosclerosis. Circulation, 2019; 139: 295-298

8) Koizumi A, Kobayashi H, Hitomi T, Harada KH, Habu T, Youssefian S: A new horizon of moyamoya disease and associated health risks explored through RNF213. Environ Health Prev Med, 2016; 21: 55-70

9) Chung J-W, Kim DH, Oh MJ, Cho YH, Kim EH, Moon GJ, Ki C-S, Cha J, Kim KH, Jeon P, Yeon JY, Kim G-M, Kim J-S, Hong SC, Bang OY: Cav-1 (caveolin-1) and arterial remodeling in adult moyamoya disease. Stroke, 2018; 49: 2597-2604

10) Hongo $H$, Miyawaki $S$, Imai $H$, Shinya $Y$, Ono $H$, Mori H, Nakatomi H, Kunimatsu A, Saito N: Smaller outer diameter of atherosclerotic middle cerebral artery associated with RNF213 c. $14576 \mathrm{G}>\mathrm{A}$ variant (rs112735431). Surg Neurol Int, 2017; 8: 104

11) Choi E-H, Lee H, Chung J-W, Seo W-K, Kim G-M, Ki C-S, Kim Y-C, Bang OY: Ring finger protein 213 variant and plaque characteristics, vascular remodeling, and hemodynamics in patients with intracranial atherosclerotic stroke: a high-resolution magnetic resonance imaging and hemodynamic study. J Am Heart Assoc, 2019; 8: e011996

12) Qiao Y, Anwar Z, Intrapiromkul J, Liu L, Zeiler SR, Leigh R, Zhang Y, Guallar E, Wasserman BA: Patterns and implications of intracranial arterial remodeling in stroke patients. Stroke, 2016; 47: 434-440

13) Kim HJ, Choi E-H, Chung J-W, Kim J-H, Kim YS, Seo W-K, Kim G-M, Bang OY: Role of the RNF213 variant in vascular outcomes in patients with intracranial atherosclerosis. J Am Heart Assoc, 2021; 10: e017660

14) Research Committee on the Pathology and Treatment of Spontaneous Occlusion of the Circle of Willis, Health Labour Sciences Research Grant for Research on Measures 
for Intractable Diseases: Guidelines for diagnosis and treatment of moyamoya disease (spontaneous occlusion of the circle of Willis). Neurol Med Chir (Tokyo), 2012; 52 : 245-266

15) Saito S, Shinmyozu K, Kawakami D, Yamauchi M, Ikeda S, Hattori Y, Yamamoto R, Hayakawa N, Ihara M: Conversion from cilostazol to OPC - 13015 linked to mitigation of cognitive impairment. Alzheimer's Dement Transl Res Clin Interv, 2021; 7: 1-9

16) Weimar C, Diener H-C, Alberts MJ, Steg PG, Bhatt DL, Wilson PWF, Mas J-L, Röther J, REduction of Atherothrombosis for Continued Health Registry Investigators: The Essen stroke risk score predicts recurrent cardiovascular events: a validation within the REduction of Atherothrombosis for Continued Health (REACH) registry. Stroke, 2009; 40: 350-354

17) D’Agostino RB, Vasan RS, Pencina MJ, Wolf PA, Cobain M, Massaro JM, Kannel WB: General cardiovascular risk profile for use in primary care: the Framingham Heart Study. Circulation, 2008; 117: 743-753

18) Nishimura K, Okamura T, Watanabe M, Nakai M, Takegami M, Higashiyama A, Kokubo Y, Okayama A, Miyamoto Y: Predicting coronary heart disease using risk factor categories for a Japanese urban population, and comparison with the framingham risk score: the Suita study. J Atheroscler Thromb, 2014; 21: 784-798

19) CAPRIE Steering Committee: A randomised, blinded, trial of clopidogrel versus aspirin in patients at risk of ischaemic events (CAPRIE). Lancet, 1996; 348: 13291339

20) Kokubo Y, Kamide K, Okamura T, Watanabe M, Higashiyama A, Kawanishi K, Okayama A, Kawano Y: Impact of high-normal blood pressure on the risk of cardiovascular disease in a Japanese urban cohort: the Suita study. Hypertension, 2008; 52: 652-659

21) Kamimura $T$, Okazaki $S$, Morimoto $T$, Kobayashi $H$, Harada K, Tomita T, Higashiyama A, Yoshimoto T, Takahashi JC, Nakagawara J, Koga M, Toyoda K, Maruyama H, Koizumi A, Ihara M: Prevalence of RNF213 p.R4810K variant in early-onset stroke with intracranial arterial stenosis. Stroke, 2019; 50: 1561-1563

22) Jackson R, Lawes CMM, Bennett DA, Milne RJ, Rodgers
A: Treatment with drugs to lower blood pressure and blood cholesterol based on an individual's absolute cardiovascular risk. Lancet, 2005; 365: 434-441

23) D’Agostino RB, Grundy S, Sullivan LM, Wilson P, CHD Risk Prediction Group: Validation of the Framingham coronary heart disease prediction scores: results of a multiple ethnic groups investigation. JAMA, 2001; 286: 180-187

24) Ueshima H, Sekikawa A, Miura K, Turin TC, Takashima N, Kita Y, Watanabe M, Kadota A, Okuda N, Kadowaki T, Nakamura Y, Okamura T: Cardiovascular disease and risk factors in Asia: a selected review. Circulation, 2008; 118: 2702-2709

25) Go AS, Chertow GM, Fan D, McCulloch CE, Hsu C-Y: Chronic kidney disease and the risks of death, cardiovascular events, and hospitalization. N Engl J Med, 2004; 351: 1296-1305

26) Hirai H, Asahi K, Yamaguchi S, Mori H, Satoh H, Iseki K, Moriyama T, Yamagata K, Tsuruya K, Fujimoto S, Narita I, Konta T, Kondo M, Shibagaki Y, Kasahara M, Watanabe T, Shimabukuro M: New risk prediction model of coronary heart disease in participants with and without diabetes: Assessments of the Framingham risk and Suita scores in 3-year longitudinal database in a Japanese population. Sci Rep, 2019; 9: 2813

27) Scholz B, Korn C, Wojtarowicz J, Mogler C, Augustin I, Boutros M, Niehrs C, Augustin HG: Endothelial RSPO3 controls vascular stability and pruning through noncanonical WNT/Ca(2+)/NFAT signaling. Dev Cell, 2016; 36: 79-93

28) Sugihara M, Morito D, Ainuki S, Hirano Y, Ogino K, Kitamura A, Hirata H, Nagata K: The AAA+ ATPase/ ubiquitin ligase mysterin stabilizes cytoplasmic lipid droplets. J Cell Biol, 2019; 218: 949-960

29) Takeda M, Tezuka T, Kim M, Choi J, Oichi Y, Kobayashi H, Harada KH, Mizushima T, Taketani S, Koizumi A, Youssefian S: Moyamoya disease patient mutations in the RING domain of RNF213 reduce its ubiquitin ligase activity and enhance $\mathrm{NF} \kappa \mathrm{B}$ activation and apoptosis in an $\mathrm{AAA}+$ domain-dependent manner. Biochem Biophys Res Commun, 2020; 525: 668-674 